\title{
Substrate noise isolation improvement in a single-well standard CMOS process
}

\author{
P. Mendonça dos Santos ${ }^{* \&}$, pedro.santos@1x.it.pt \\ Luís Mendes ${ }^{* \pm}$, lmendes@ipleiria.pt \\ João Caldinhas Vaz ${ }^{* \#}$, joaovaz@ tecnico.ulisboa.pt \\ *Instituto de Telecomunicações, Lisboa, Portugal, \& Academia Militar, Lisboa, Portugal, ${ }^{ \pm}$Instituto Politécnico de \\ Leiria, Leiria, Portugal, "Instituto Superior Técnico, Universidade de Lisboa, Lisboa, Portugal
}

Corresponding author - Pedro Mendonça dos Santos; phone: (+351)218418388; e-mail: pedro.santos@1x.it.pt

Instituto de Telecomunicações/IST, Av. Rovisco Pais, 1049-001, Lisboa, Portugal.

\begin{abstract}
This work describes a fully CMOS compatible methodology, which makes available a pseudo deep n-well in single-well standard CMOS process. The proposed method is based on mask manipulation to accommodate the field implant p-type region into the n-well, and does not require any additional masks or modification in the CMOS process flow. According to the experimental results, the floating NMOS made available by the methodology shows a reduction in the threshold voltage, which implies a slight improvement in its performance, when compared with its standard NMOS counterpart. It was also experimentally demonstrated up to 3 $\mathrm{GHz}$, that the guard-ring field implant/pseudo deep n-well proposed structure improves substrate noise isolation when compared to the classical $\mathrm{p}+$ guard-ring, with a maximum improvement above $20 \mathrm{~dB}$ for low frequencies and a minimum of $4 \mathrm{~dB}$ at $3 \mathrm{GHz}$.
\end{abstract}

Keywords - CMOS technology, MOSFET, Integrated circuit noise.

\section{INTRODUCTION}

As CMOS technology continues its scaling down to the nanometer range, processing is also becoming more complex, with an increasing number of masks and related processing steps. Modern ICs include a diversity of digital, analog, mixed-signal and radio-frequency (RF) functional blocks, with different power, bias levels and signals of different frequencies, sharing the same CMOS die. Also, very high frequency digital and power management parts are responsible for the increase of the noise level in the $\mathrm{IC}$, which can seriously affect the performance of sensitive analog and RF parts, like band-gap references, low-noise amplifier and oscillator circuits.

To circumvent these problems, CMOS technology has evolved, not only above Silicon (e.g., thinner dielectrics, low and high $\mathrm{k}$ dielectrics, increased number of metal levels and accessibility to thick Copper layers), but also at Silicon level, with the availability of trench isolation structures, multiple well processes and, in some specific cases, buried layers.
CMOS processes including a deep n-well are available to circuit designers from, at least, a decade ago [1]. Some works report that deep n-well regions can be used to improve substrate noise isolation [1]-[6] and devices performance, namely, increase of the cutoff and maximum oscillation frequencies [1], [6], and reduction of harmonic distortion [7]. Furthermore, deep wells are widely used in the development of high-voltage (HV) devices in HV CMOS [8]-[11] and in CMOS compatible photodiode and phototransistors structures, aimed at optical and communication applications [12]- [14].

For some applications, where layout area is not the major concern, designers can still resort to 'older' technology nodes (> $180 \mathrm{~nm}$ ), if functionality versus Silicon footprint trade off becomes acceptable, especially at the prototyping stage. However, the deep n-well formation is not available in those CMOS processes. In that case, circuit design could become unviable if applications requiring different reference levels and ground floating devices are in scope.

In this work a fully CMOS compatible methodology is used to create a p-type isolated region inside the single n-well, resorting to the p-type field implantation available in the process. The idea is to adapt the concept of the deep n-well and bring its advantages to single-well CMOS processes, with the associated advantages, like the availability of floating circuits (different references, not connected to the die p-type substrate), and correlated substrate noise cross-talk reduction. In this way, these processes, which are poor in features when compared to recent nodes with multiple wells, becomes enriched in functionality.

Section 2 describes the mask manipulation methodology to achieve the isolated lightly doped p-type region inside the standard n-well, without any modification in the fabrication steps of the CMOS process. In Section 3 the proposed methodology is validated throughout experimental results. In Section 4, experimental results also demonstrate that these field implanted isolated regions can be used to significantly reduce substrate noise propagation. Finally, in section 5, some conclusions are drawn. 


\section{THE DESIGN OF THE ISOLATED P-TYPE REGION}

The complete envelopment of a lightly doped p-type region inside an n-well region is an obvious task in a triple well CMOS process, due to the availability of the deep n-well. In these cases, the isolated region will be formed by a p-well implant over the pre-processed deep n-well [1], [6], [8], [10][11].

In single well CMOS processes this possibility is not considered. In fact, basic CMOS processing is founded on two basic requirements: a p-type substrate (p-sub) that forms the bulk of the NMOS transistors; and the presence of an n-well to act as the local bulk for the complementary PMOS transistors. Fig. 1 a) shows the part of the typical mask arrangement used for the layout design of the complementary pair $(\mathrm{n}+, \mathrm{p}+$ and contact masks not shown, for simplicity). In general, a shallow $\mathrm{p}$-type region is formed in all those regions where $\mathrm{n}$-well is not present. This p-type implant is usually denoted as field implant or P-block region, and it serves, not only to increase parasitic devices threshold voltage, but also to increase the bulk concentration of the standard NMOS transistors. This region is always shallower than the n-well. Depending on each CMOS processing rules, this p-type region is usually defined by an automatic mask generation algorithm, in order to be implanted wherever n-well is absent. For the CMOS process described in Fig. 1 a), the automatic mask tool will generate a field implant layout layer (FIMP) coincident with the n-well layout layer (NW). Since the former has a dark field mask, field implantation will only be processed in regions not covered by the field implant layout layer, i.e., those regions without $n$ well. Similar relations between these layers can be found for the case of other CMOS processes.

This work proposes and discusses the validity of implanting a field implant region with its volume completely surrounded by the $\mathrm{n}$-well pre-processed region. Taking into account that field implant is shallow than the n-well, it is possible to consider its complete immersion in the n-well, as long as the correspondent layout layers are manipulated accordingly. The proposed mask modification is depicted in Fig. 1 b). The figure shows that a field implant mask-opening (NOT[FIMP]) window is cut and left covered by the same n-well mask layout. During processing, after n-well formation according to the NW layer, this opening in the field implant mask will allow acceptor ion penetration only in the (NOT[FIMP]) defined window, forming an isolated lightly doped p-type volume. As can be seen, this p-type volume is completely enclosed by the $\mathrm{n}$-well volume, which can be considered as a pseudo deep n-well.

Fig. 1 b) shows the implementation of a floating NMOS in this p-type isolated region, showing the junction isolation between the n-well and the p-field (at left) to form the proposed floating NMOS.

This work gives particular emphasis to the designer point of view. In fact, for fabless design houses, or in the case of research works, where prototyping cost is critical, this methodology can overcome the necessity for extra masks or an expensive CMOS process. Like in CMOS processes with an available deep n-well, this proposal permits the fabrication of complete and isolated analog, digital or mixed mode circuits inside this pseudo deep n-well. The methodology is only appropriate to single-well CMOS processes and becomes, of course, superfluous in processes where the deep n-well is already available as a regular mask.

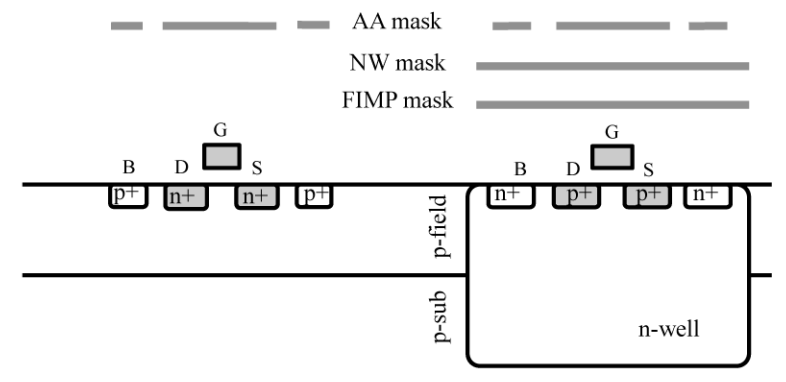

a)

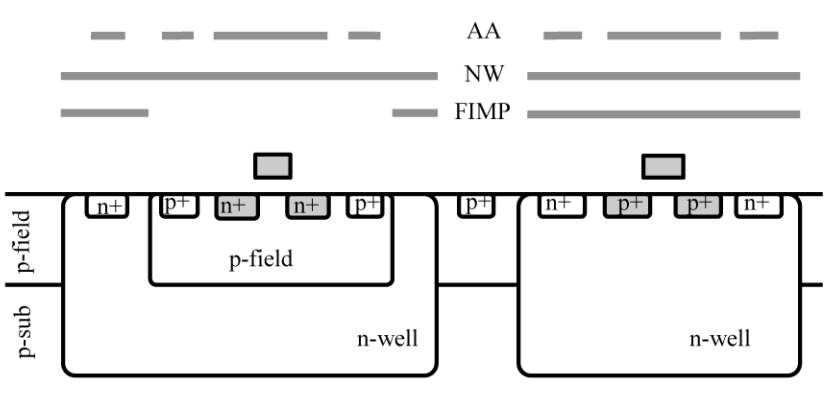

b)

Fig. 1 - Cross sections of the structures under study, showing the proposed masks arrangements: a) The conventional NMOS/PMOS complementary pair; b) the "opening" in the FIMP mask layer (left) for the formation of a field implant region inside the pre-processed n-well, to accommodate a floating NMOS.

\section{STANDARD AND FLOATING NMOS CHARACTERIZATION}

One of the objectives of the proposed methodology is to achieve a floating NMOS device, with its bulk formed by the p-type field implant present in the CMOS process. The viability of designing such MOSFET and the evaluation of its performance is discussed in this Section. For comparison purposes two geometrically identical NMOS devices were designed and fabricated in a single-well standard CMOS process. Both devices have a channel width and length of $\mathrm{W}=5 \mu \mathrm{m}$ and $\mathrm{L}=0.35 \mu \mathrm{m}$, respectively. Besides the differences in the field implant (FIMP) and active area (AA) masks, the remaining masks (NW, $\mathrm{n}+$ implants, contacts and metal interconnections) were kept exactly identical. The characterization of both devices was achieved with an Agilent 4156C Semiconductor Parameter Analyzer.

Fig. 2 shows the experimental $\mathrm{I}_{\mathrm{D}}\left(\mathrm{V}_{\mathrm{GS}}\right)$ characteristics, obtained with $\mathrm{V}_{\mathrm{DS}}=0.1 \mathrm{~V}$, for the standard NMOS and the proposed floating NMOS. It can be noticed that the floating NMOS current is higher than the standard NMOS current, even if this difference is almost negligible at strong inversion. However, in the subthreshold region, devices characteristics differences are significant. This difference is also relevant at cutoff, when the samples are exposed to light. In these conditions, the 
leakage current for $\mathrm{V}_{\mathrm{GS}}<0 \mathrm{~V}$ present a flat, $\mathrm{V}_{\mathrm{GS}}$ independent, behavior, but the value in the standard NMOS is almost a decade above the proposed floating NMOS. For the die samples measured in dark, both devices have leakage currents below the sensitivity level of the experimental apparatus $(<1$ $\mathrm{pA})$.

These differences in the subthreshold region can be explained by the change in the net doping concentration and resultant doping profile when a lightly doped p-type implant is processed over a lightly doped n-type region, the n-well, instead of the standard p-type substrate of the chip. These different performances of the devices under comparison will be discussed in the following subsections.

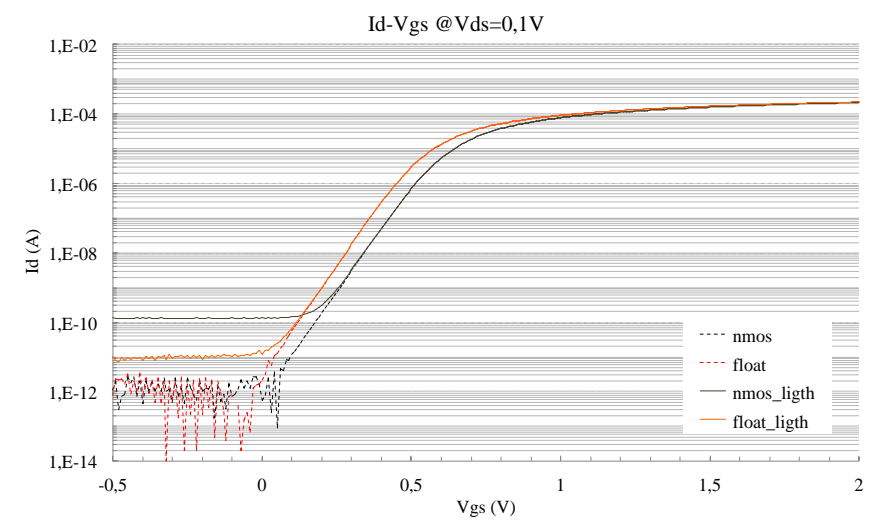

Fig. 2 - Comparative experimental $\mathrm{I}_{\mathrm{D}}\left(\mathrm{V}_{\mathrm{GS}}\right)$ characteristics, for $\mathrm{V}_{\mathrm{DS}}=0.1 \mathrm{~V}$, with the die sample in dark and with direct light.

\subsection{On the difference in threshold voltages}

As mentioned before, the main physical structural difference between the standard and the proposed floating NMOS is in the bulk of the devices. In the standard NMOS, the surface channel doping is the resultant net doping of the constant $\mathrm{p}$ type substrate of the die added by the field implant and the subsequent NMOS p-type threshold adjust implant concentrations.

Fig. 3 [15] shows the doping profile (black line) of the CMOS process field region obtained with Secondary Ion Mass Spectrometry (SIMS), showing the constant concentration of the substrate $\left(\sim 6 \times 10^{14} \mathrm{~cm}^{-3}\right)$, over which two heavier p-type doped regions are formed: the field implant, with a peak concentration at the surface around $6 \times 10^{16} \mathrm{~cm}^{-3}$ and a 'Gaussian like' decay up to $3.5 \mu \mathrm{m}$ depth; and the shallow threshold adjust implant, with a peak concentration below $3 \times 10^{17} \mathrm{~cm}^{-3}$. The standard NMOS doping profile at the channel is identical to the one represented in Fig. 3, except that the origin, i.e. the $\mathrm{Si}-\mathrm{SiO}_{2}$ interface, is shifted to the threshold voltage adjust peak concentration, due to the mask effect of the Poly-Si gate in this implantation step.

It is well known that the threshold voltage has an explicit dependence on the channel concentration. For a non-uniform profile with multiple implants, like the one depicted in Fig. 3, one can use a step approximation to define the high-low profile in the channel (dots in Fig. 3), with a step value of $\mathrm{N}_{\mathrm{A}}=4 \times 10^{16} \mathrm{~cm}^{-3}$ at the surface. In sub threshold, i.e., for very low $\mathrm{V}_{\mathrm{GS}}$ and $\mathrm{V}_{\mathrm{DS}}$, the density of electrons at the surface is

$n_{s}=n_{i} \mathrm{e}^{\mathrm{q}\left(\psi_{s}-\psi_{B}\right) / \mathrm{kT}}$

where $\mathrm{n}_{\mathrm{i}}$ is the intrinsic carrier concentration $\left(\mathrm{cm}^{-3}\right), \mathrm{q}$ is the elementary charge, $\psi_{S}$ is the surface potential, $\psi_{B}$ is the difference between Fermi and intrinsic levels $\left(\mathrm{E}_{\mathrm{F}}-\mathrm{E}_{\mathrm{i}} / \mathrm{q}\right), \mathrm{k}$ is the Boltzmann constant, and $\mathrm{T}$ is the temperature.

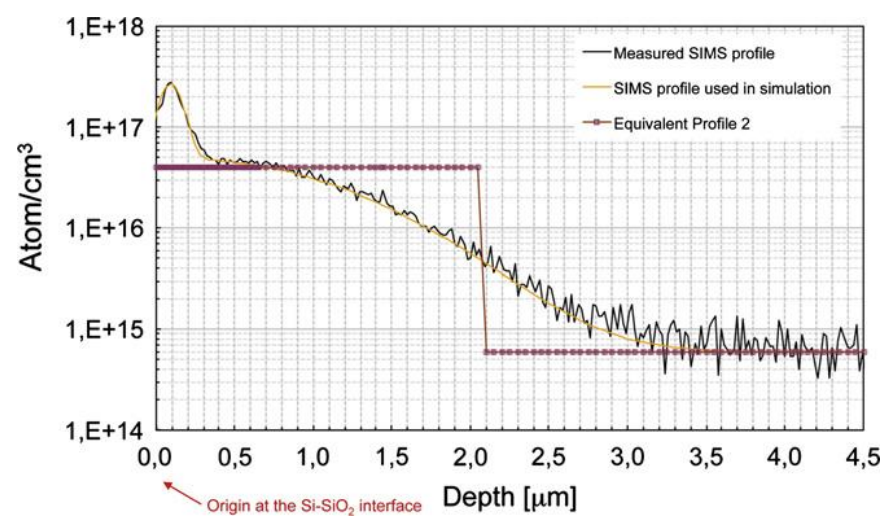

Fig. 3 - CMOS process field region doping profile, obtained with SIMS (from the author work, in [15]).

At the onset of inversion, the electron concentration at the surface is $\mathrm{n}_{\mathrm{s}}=\mathrm{N}_{\mathrm{A}}$, and

$N_{A}=n_{i} \mathrm{e}^{\mathrm{q}\left(\psi_{s}-\psi_{B}\right) / \mathrm{kT}}$

which can be solved for $\psi_{S}-\psi_{B}$. At the onset of strong inversion, one can assume that $\psi_{S}=2 \psi_{B}$, and the surface potential for inversion can now be derived,

$\psi_{s}(i n v)_{n m o s}=\frac{2 k T}{q} \ln \left(\frac{N_{A}}{n_{i}}\right)$

The threshold voltage of the standard NMOS device $\left(V_{\text {Tnmos }}\right)$ can then be derived by the well-known following equation:

$V_{\text {Tnmos }}=V_{f b}+\psi_{s}(\mathrm{inv})+\frac{\sqrt{2 \varepsilon_{S} q N_{A}\left(\psi_{S}(\mathrm{inv})-V_{S B}\right)}}{\operatorname{Cox}}$

where $V_{f b}$ is the flat-band voltage shift, that includes the influence of the Poly-Si gate and semiconductor work function difference, and the effects of oxide charges and interface traps; $\mathrm{V}_{\mathrm{SB}}$ is the source-bulk applied voltage, which is zero in this work; and $\mathrm{C}_{\mathrm{ox}}$ is the CMOS process gate-oxide capacitance per area. 
In the case of the floating NMOS, the N-well implantation step appears as a compensation doping 'behind' the subsequent p-field implant (FIMP). In this case, again assuming a step approximation for the n-well donors concentration, $\mathrm{N}_{\mathrm{D}}$, the hole surface concentration at flat-band condition would be $\mathrm{p}=\mathrm{N}_{\mathrm{A}}-\mathrm{N}_{\mathrm{D}}$.

According to the mentioned above for the standard NMOS, the onset of inversion for the floating device can be achieved when $n_{s}=N_{A}-N_{D}$, and the surface potential for inversion is now

$\psi_{s}(i n v)_{\text {float }}=\frac{2 k T}{q} \ln \left(\frac{N_{A}-N_{D}}{n_{i}}\right)$

The floating device threshold voltage $\left(V_{T \text { float }}\right)$, assuming also $\mathrm{V}_{\mathrm{SB}}=0$, is

$V_{\text {Tfloat }}=V_{f b_{f}}+\psi_{s}(\mathrm{inv})_{\text {float }}+\frac{\sqrt{2 \varepsilon_{s} q\left(N_{A}-N_{D}\right)\left(\psi_{s}(\mathrm{inv})_{\text {float }}\right)}}{\operatorname{Cox}}$

For the present study and under the mentioned experimental characteristics, the standard NMOS and the proposed floating NMOS are geometrically identical. According to the proposed methodology, the CMOS process steps are unaltered and the unique difference resides on mask manipulation. This means, for example, that all the implants, including field and threshold implants, are locally imposed with the same doses and energies. Furthermore, it can be assumed that $\mathrm{V}_{\mathrm{fb}}$ is identical in both NMOS, since oxide growth and gate processing are unaltered. Thus, the difference in the devices threshold voltages, $\Delta V_{T}$, can be derived from the difference between equations (2) and (4),

$\Delta V_{T}=\psi_{s}(\mathrm{inv})_{n m o s}+\frac{\sqrt{2 \varepsilon_{s} q N_{A}\left(\psi_{s}(\mathrm{inv})-V_{S B}\right)}}{\operatorname{Cox}}-\left(\psi_{s}(\mathrm{inv})_{\text {float }}+\right.$

$\left.\frac{\sqrt{2 \varepsilon_{s} q\left(N_{A}-N_{D}\right)\left(\psi_{s}(\mathrm{inv})_{\text {float }}\right)}}{\operatorname{Cox}}\right)$

where it was assumed that both terms of $V_{\text {fb }}$ were canceled.

Using (1) and (3) in Equation (5) and after simplification, the threshold voltage difference can be evaluated by the following equation, as a function of the n-well doping concentration $\left(\mathrm{N}_{\mathrm{D}}\right)$,

$\Delta V_{T}\left(N_{D}\right)=$

$\frac{2 k T}{q}\left(\ln \frac{N_{A}}{N_{A}-N_{D}}\right)+\frac{\sqrt{4 \varepsilon_{S} k T}}{\operatorname{Cox}}\left(\sqrt{N_{A} \ln \frac{N_{A}}{n_{i}}}-\sqrt{\left.\left(N_{A}-N_{D}\right) \ln \frac{N_{A}-N_{D}}{n_{i}}\right)}\right.$

which becomes zero in the absence of the n-well $\left(\mathrm{N}_{D}=0\right)$.

Fig. 4 shows the variation of the threshold voltage difference with the step n-well concentration, for an approximate floating surface concentration of $\mathrm{N}_{\mathrm{A}}=4 \times 10^{16} \mathrm{~cm}^{-3}$. As can be seen, the threshold voltage difference increases with the doping level of the n-well. For $\mathrm{N}_{\mathrm{D}}$ below a doping concentration of $10^{15} \mathrm{~cm}^{-3}$, the difference in threshold voltages is negligible $(\sim 1.3 \mathrm{mV})$. It should be pointed out that equation (6) is only valid for $\mathrm{N}_{\mathrm{D}}<\mathrm{N}_{\mathrm{A}}$, corresponding to a resultant p-type floating NMOS bulk. Otherwise, if the number of donors' impurities overcomes the acceptor concentration, the bulk would be ntype and the experimental device would not be functional.

Taking as reference the difference of approximately $60 \mathrm{mV}$ from the data presented in Fig. 2, results in an n-well concentration around $2.7 \times 10^{16} \mathrm{~cm}^{-3}$. This donor concentration acts as a counter doping, reducing the overall net doping at the surface and increasing the available electrons at the surface in weak inversion, for the same $\mathrm{V}_{\mathrm{GS}}$ voltage.

According to the experimental data shown in Fig. 2, this threshold voltage difference is patent in the subthreshold region. This difference is maintained even at strong inversion, but with less impact. In fact, for $V_{G S}>>V_{T}$, the resultant overdrive voltage $\left(\mathrm{V}_{\mathrm{GS}}-\mathrm{V}_{\mathrm{T}}\right)$ is less relevant in the devices performance. This can be confirmed in the experimental static characteristics for the standard and the floating devices, shown in Fig. 5. As can be seen, in the $\mathrm{I}_{\mathrm{D}}\left(\mathrm{V}_{\mathrm{DS}}\right)$ graphic, for the same $\mathrm{V}_{\mathrm{GS}}$ value, saturation current is always higher for the proposed floating NMOS.

\subsection{On the leakage difference at cutoff}

Getting back to the comparative curves in Fig. 2 (dark mode), and for very low and negative $\mathrm{V}_{\mathrm{GS}}$, devices drain current is not measurable, due to the experimental setup 'noise level' $(\sim 1$ $\mathrm{pA})$. In these cases the samples were tested with the probe station microscope light turned off.

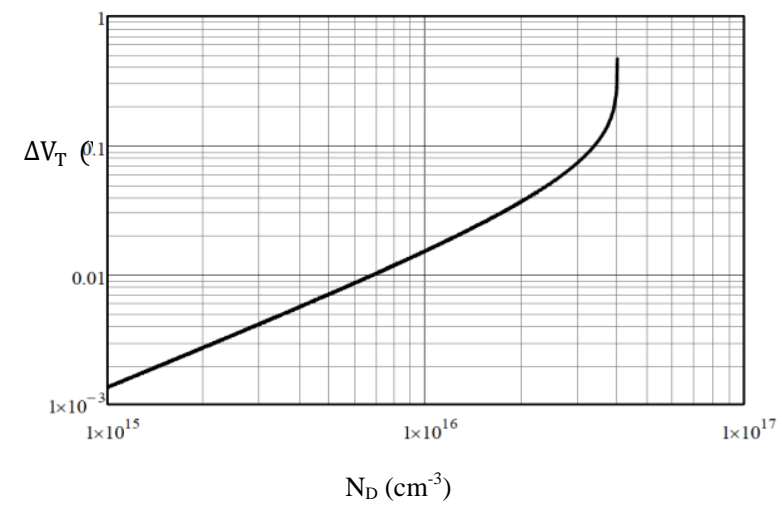

Fig. 4 - Variation of the threshold voltage difference between the devices under study, with the n-well concentration, $\mathrm{N}_{\mathrm{D}}$, for $\mathrm{N}_{\mathrm{A}}=4 \times 10^{16} \mathrm{~cm}^{-3}$.

It was experimentally found that the probe station microscope light has a considerable influence in the leakage current levels. As can be confirmed in Fig. 2, when the samples were submitted to an equal light flux, both the floating and the standard NMOS experience a flat, $\mathrm{V}_{\mathrm{GS}}$ independent, characteristic, as $\mathrm{V}_{\mathrm{GS}}$ becomes very low and negative. However, this leakage drain current is almost a decade lower for the floating device case. Further experiments were accomplished in order to understand this detail. 


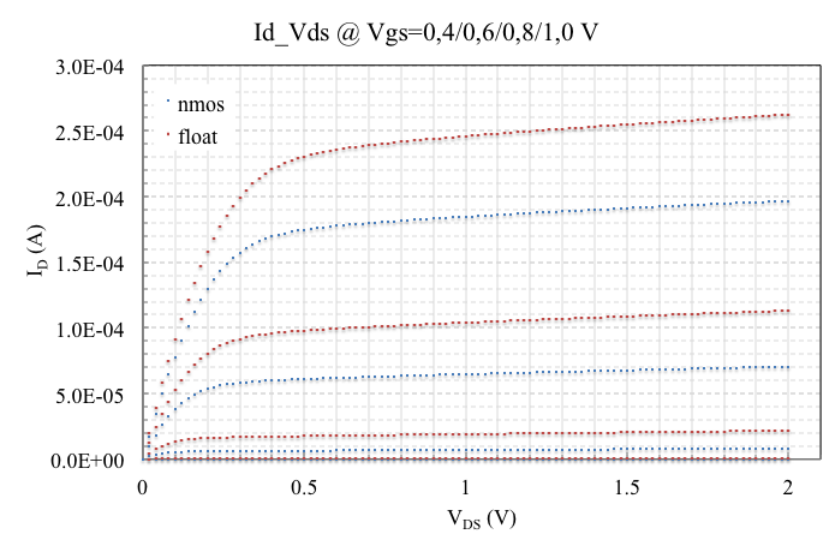

Fig. 5 - Comparative experimental $\mathrm{I}_{\mathrm{D}}\left(\mathrm{V}_{\mathrm{DS}}\right)$ for $\mathrm{V}_{\mathrm{GS}}$ values of $0.4 \mathrm{~V}$ to $1 \mathrm{~V}$ (in $0.2 \mathrm{~V}$ steps).

Fig. 6 shows the experimental comparative characteristics of the standard and floating NMOS, with the gate floating, and with the bulk-source junction grounded. This arrangement behaves like an NPN bipolar transistor, with the collector acting as the drain, the source as the emitter and the base as the bulk. For this transistor, the base-emitter junction is shortcircuited to ground. Both devices were tested in these conditions under dark, medium and full light microscope intensity.

As can be seen in Fig. 6, without light, both collector (drain) currents are at the level of the apparatus sensitivity, and no conclusion can be drawn about the differences between devices leakage current levels. For the medium level of light intensity, a photo-generated current appears at the collector, represented by the increase in the collector current for reverse collector-base (drain-bulk) junction biasing. Like in Fig. 2, this current is independent of $\mathrm{V}_{\mathrm{CB}}$, confirming that the extra carriers are generated by the presence of light. At this level of illumination, it is clear that the NMOS floating device leakage current is more than a decade lower than the correspondent current in the standard NMOS. Furthermore, when both devices are exposed to the maximum light intensity, photo generated current increases by the same order of magnitude in both devices, which confirms that this generation process is independent of the proposed methodology. This leads to the conclusion that the floating NMOS has inherently a lower leakage current.

Table 1 summarizes the most relevant experimental parameters measured on both devices, showing the quantitative differences between the devices under study for threshold voltage $\left(\mathrm{V}_{\mathrm{TH}}\right)$, subthreshold slope $(\mathrm{S})$, On-resistance $\left(\mathrm{R}_{\mathrm{ON}}\right)$ and maximum transconductance $\left(\mathrm{g}_{\mathrm{m} \_\max }\right)$. As can be seen, the floating NMOS presents improved specifications in all parameters, due to the resultant net doping difference in the bulk.

\section{IMPROVING NOISE ISOLATION}

The results discussed in the previous section validate the methodology and the fabrication viability of floating NMOS transistors, isolated from the substrate die, which are found very useful for some mixed mode and RF applications [1], [6][7], [16]. Besides that, literature also shows that deep n-wells used in CMOS technology improves cross talk isolation and reduces substrate noise [2]-[3], [16]. For these reasons, it is mandatory to evaluate these characteristics for the proposed pseudo deep n-well structure. Accordingly, a set of test structures was designed and fabricated, in order to estimate the level of noise and interference immunity for circuits operating inside the pseudo deep n-well. The level of isolation provided to the neighboring circuits, if a noisy circuit is working inside the proposed structure, was also investigated.

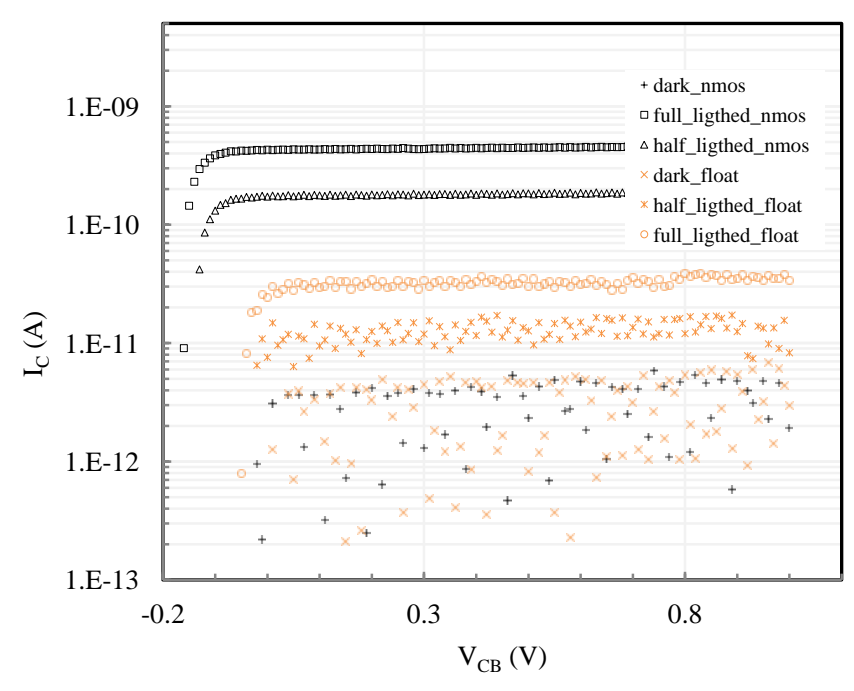

Fig. $6-\mathrm{I}_{\mathrm{C}}$ current vs. $\mathrm{V}_{\mathrm{CB}}$ voltage for three different levels of light intensity, for the standard and the floating NMOS devices.

Table 1

Experimental parameters for the devices under comparison.

\begin{tabular}{lcccc}
\hline NMOS & $\mathrm{V}_{\mathrm{TH}}(\mathrm{V})^{1}$ & $\mathrm{~S}(\mathrm{mV} / \mathrm{dec})$ & $\mathrm{R}_{\mathrm{ON}}(\Omega)^{2}$ & $\mathrm{~g}_{\mathrm{m} \_\max }(\mu \mathrm{A} / \mathrm{V})$ \\
Standard & 0.54 & 83 & 1286 & 206 \\
Floating & 0.48 & 81 & 1098 & 209 \\
\hline \multicolumn{5}{c}{${ }^{1} \mathrm{~V}_{\mathrm{DS}}=0.1 \mathrm{~V} ;{ }^{2} \mathrm{~V}_{\mathrm{DS}}=0.1 \mathrm{~V}, \mathrm{~V}_{\mathrm{GS}}=1 \mathrm{~V}}$.
\end{tabular}

Fig. 7 a) shows a microphotograph of the fabricated test structures. Some of them are for de-embedding purposes due to the high frequency measurements. All the test structures were designed with an identical mask layout arrangement above Silicon (pre and inter metal dielectrics and metallization layers), including the three pads arrangements to be accessed by ground-signal-ground (GSG) probes.

The level of substrate noise isolation was measured with the aid of the test structures represented in Figs. 7 b) to d). The reference structure is composed by two p+implants separated by $20 \mu \mathrm{m}$, as illustrated in Fig. 7 b). Fig. 7 c) shows the inclusion of a classical $p+$ guard-ring surrounding the $p+$ pick up at left. Fig. 7 d) is the cross section of the proposed isolation structure, showing the complete immersion of the field and p+ pick up implants in the pseudo deep n-well. In 
this structure, the n-well is grounded to ensure that the obtained isolation is always the worst as possible. It must be noted that the $20 \mu \mathrm{m}$ distance between the $\mathrm{p}+$ noise emitter $(\mathrm{N}$, at right) and the pick-up $\mathrm{p}+(\mathrm{S}$, at left) implants was kept constant in all test structures. In practice, to maintain the coherence in this comparative study, layout areas for the investigated structures were kept equal, in order to properly evaluate the isolation versus Silicon footprint trade off.

These structures were experimentally characterized with an Agilent E8361A Vector Network Analyzer (VNA), up to 3 $\mathrm{GHz}$, in order to quantify each level of isolation. The GSG probes used are from Cascade Microtech and are proper for measurements in the $\mathrm{GHz}$ range. The test equipment (VNA and probes) was calibrated using an impedance standard substrate provided by the probes manufacturer.

Fig. 8 shows the measured transmission scattering parameter (S21) for the test structures outlined in Figs. 7 b) to d). As can be seen, the standard CMOS field implant over p-sub provides about $30 \mathrm{~dB}$ of signal attenuation. The figure also shows that the isolation is almost constant in frequency, increasing only 3 $\mathrm{dB}$ in the measurement frequency range. The level of attenuation of the reference structure (Fig. 7 b)) has an increase of $30 \mathrm{~dB}$ when the pick up is surrounded by a classic p+ guard-ring structure, like the one of Fig. $7 \mathrm{c}$ ). Besides that, the graphic in Fig. 8 shows that the frequency behavior of the $\mathrm{p}+$ classic guard-ring structure is similar to the one of the reference structure. The increase of attenuation verified between the first two structures is due to the fact that the $\mathrm{p}+$ regions act as noise or interference drains, reducing, in this way, the noise or interference that propagates in the field implant region and that reaches the $\mathrm{p}+$ pick up region $(\mathrm{S})$.

Fig. 8 also shows that the attenuation provided by the proposed isolation structure is the highest achieved for the measurement frequency range $(<3 \mathrm{GHz})$. For the lower end of the measurement frequency band (few hundreds of $\mathrm{MHz}$ ) the attenuation reaches the VNA noise level, with an attenuation of $80 \mathrm{~dB}$, which corresponds to an improvement higher than $20 \mathrm{~dB}$ in comparison with the $\mathrm{p}+$ guard-ring structure.

For higher frequencies this difference becomes lower, attaining only $5 \mathrm{~dB}$ at $3 \mathrm{GHz}$ (isolation of $65 \mathrm{~dB}$ ). This behavior deserves further discussion and a more complete investigation must be taken into account.

Fig. 7 b) shows the equivalent resistive path between the noise (N) and the pick-up (S), modeled by $\mathrm{R}_{\mathrm{FB}}$. In Fig. $7 \mathrm{c}$ ), it can be initially assumed that $\mathrm{R}_{\mathrm{FB}}$ could be equivalent to $\left(\mathrm{R}_{\mathrm{FL}}+\mathrm{R}_{\mathrm{p}}+2 \mathrm{R}_{\mathrm{F}}\right)$. However, the experimental results depicted in Fig. 8, clearly indicates that the influence of the second $R_{F}$ resistive path, between the first $\mathrm{p}+$ guard-ring and the sense pick-up (S), is severely reduced (almost 30dB). Fig. 7 d) shows the p+ pick-up implant $\mathrm{S}$ immersed in the proposed field implant/pseudo deep n-well junction isolation structure, including the parallel RC models of the FIMP/n-well junctions.

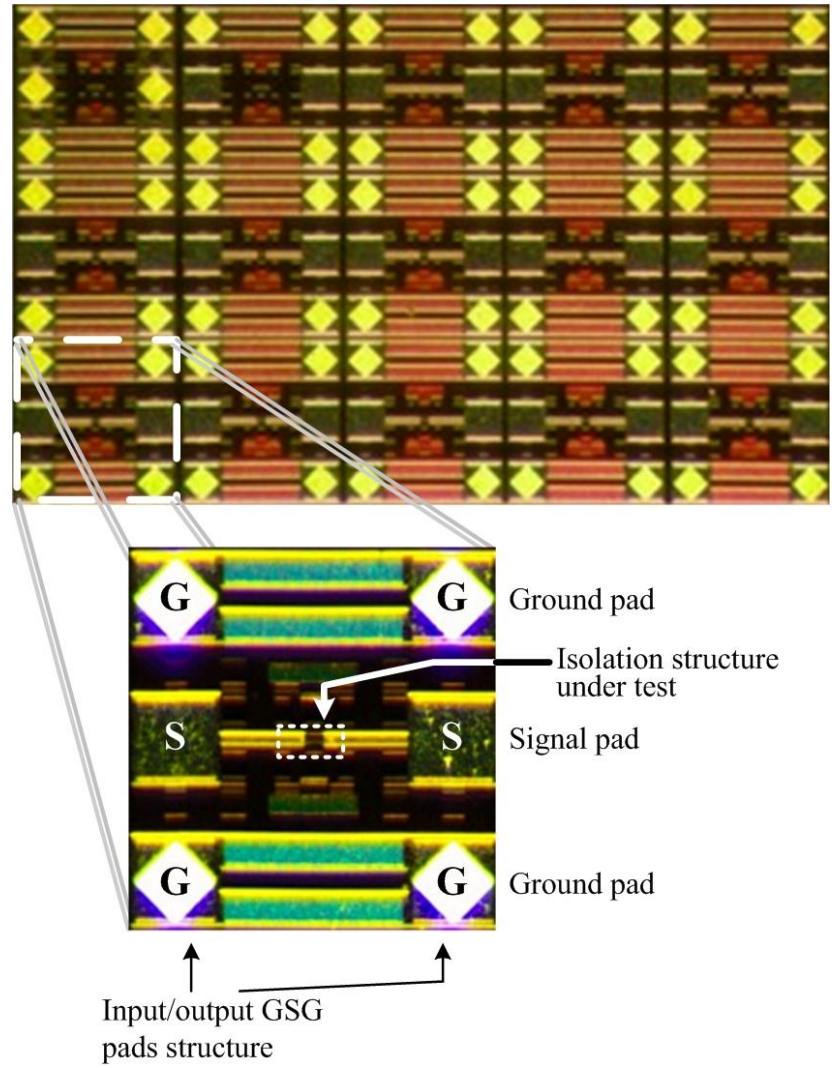

a)

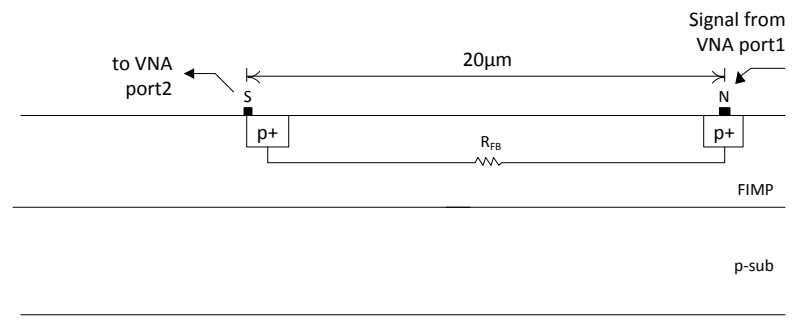

b)

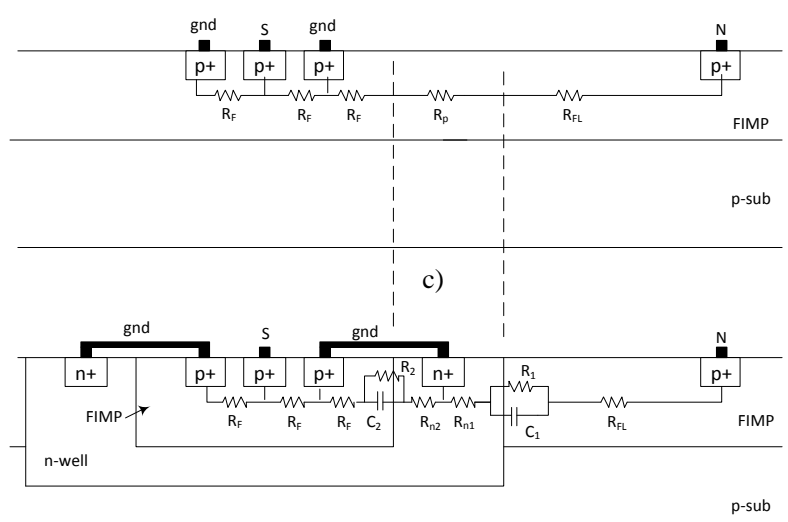

d)

Fig. 7 - Test structures to evaluate substrate isolation: a) microphotograph of the fifteen fabricated test cells, showing the detail of the GSG pad arrangement at input and output ports; b) cross section representation of the reference structure, showing the $\mathrm{p}+$ noise $(\mathrm{N})$ port, at right, and the $\mathrm{p}+$ pick-up port (S), at left, separated by $20 \mu \mathrm{m}$; c) the same structure, with a classical p+ guard-ring; d) p+ pick-up immersed in the proposed isolation structure (note: cross sections are to scale). 
For the three different situations (Figs. 7 b), c), and d)), the isolation is frequency dependent only when the n-well is present. This is related with the fact that the injected noise has to cross two reverse biased $\mathrm{PN}$ junctions, whose equivalent circuits are the parallel RC networks, $\mathrm{R}_{1} / / \mathrm{C}_{1}$ and $\mathrm{R}_{2} / / \mathrm{C}_{2}$.

For very low frequency values the proposed pseudo n-well structure provides high isolation when compared to the previous solutions, since the reactances $\left(\mathrm{C}_{1}\right.$ and $\left.\mathrm{C}_{2}\right)$ are almost negligible and the reverse biased $\mathrm{PN}$ junction resistive effect $\left(\mathrm{R}_{1}\right.$ and $\mathrm{R}_{2}$ in Fig. $\left.7(\mathrm{~d})\right)$ dominates with a high value.

For increasing frequency values the capacitive reactance becomes dominant over $\mathrm{R}_{1}$ and $\mathrm{R}_{2}$ and the isolation decreases with frequency. This isolation degradation will continue up to a frequency value where the noise path resistance becomes dominant over the capacitances values. This path resistance and the capacitances appear in series (assuming a straight-line 1D analysis), which means that, without considering other capacitive effects besides the junctions, the isolation will became frequency independent. This is apparent from our measurement results, although the $3 \mathrm{GHz}$ maximum frequency is not high enough to point out further effects.

For low frequencies, the reasons for an improved isolation with the structure proposed in Fig. 7 d) are the PN junctions $R_{1}$ and $R_{2}$ high values resistances and the additional $n$-well connection to ground (between $R_{n 1}$ and $R_{n 2}$ ) that further deviates the noise from reaching the pick-up point $\mathrm{S}$.

For increasing frequencies, where $\mathrm{C}_{1}$ and $\mathrm{C}_{2}$ reactance's becomes much lower than the noise path resistance, PN junctions can be considered to converge to very-low impedances. A geometric/electric comparison of the structures depicted in Fig. 7 c) and d), and the assumption that FIMP equivalent resistance outside and inside the n-well are similar, leads to the following conclusions:

- The path resistance between $\mathrm{N}$ and the first $\mathrm{PN}$ junction (modeled by $\left.\mathrm{R}_{1} / / \mathrm{C}_{1}\right)$ is equal in both cases $\left(\mathrm{R}_{\mathrm{FL}}\right)$.

- The path resistance between $\mathrm{S}$ and the second $\mathrm{PN}$ junction (modeled by $\left.\mathrm{R}_{2} / / \mathrm{C}_{2}\right)$ is also equal in both cases $\left(2 \mathrm{R}_{\mathrm{F}}\right)$.

- There will be eventually a different equivalent resistance for the paths $R_{P}$ and $R_{n 1}+R_{n 2}$, depending on the concentration differences between the n-well and the FIMP.

- The extra connection to ground in the n-well region appears as an advantage for noise path reduction.

According to these, and with the interpretation of the data in Fig. 8, it can be concluded that, even if $R_{p}>R_{n 1}+R_{n 2}$, the effect of the ground connection in the $n$-well is relevant enough to achieve better isolation in the case of the structure of Fig. $7 \mathrm{~d}$ ). This indicates that the use of a field implant region inside a pseudo deep n-well can improve wideband isolation over the classical $\mathrm{p}+$ guard-ring structure, at least for frequencies up to $3 \mathrm{GHz}$. For frequencies above the ones measured in this work, it is expected a closer performance in terms of both structures.

In future works, the discussion on the $R_{p}>R_{n 1}+R_{n 2}$ inequality can become relevant, to prove there is no overturn of isolation for the structure with the classical p+ guard-ring. It should be noted that FIMP concentration is always higher than n-well concentration. However, it is also well known that p-type $\mathrm{Si}$ is more resistive than n-type $\mathrm{Si}$ for the same carrier concentrations (for non-degenerated doping levels). Conclusions about the performance of the isolation proposal for frequencies above $3 \mathrm{GHz}$ will be object of future studies.

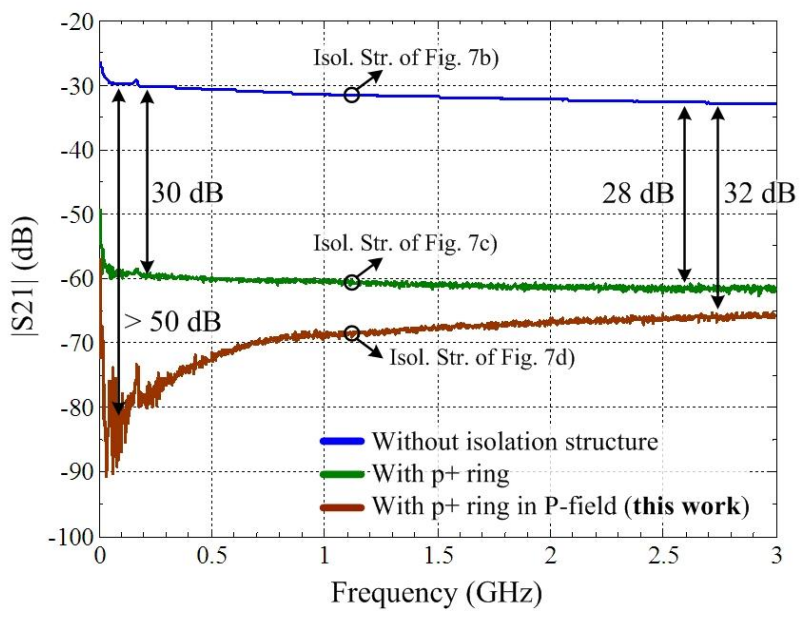

Fig. 8 - Experimental results for the S21 parameter, obtained from the test structures schematized in Figs. 7 b) to d).

It should be again emphasized that there is no process complexity increase, extra masking or Silicon area penalty, when the proposed structure of Fig. 7 d) is used to improve isolation from the substrate. PMOS and especially NMOS transistors operating inside this pseudo deep n-well should experience lower levels of noise or interferences injected from the substrate. The proposal is particularly appropriate for the case of sensitive analog and RF blocks, like voltage and current references and low-noise amplifiers and oscillators. Some situations that can also be improved are, for example, in a direct conversion fully integrated transceiver, the oscillator frequency pulling caused by the power amplifier in the emitter path or the DC offset errors due to self-mixing in the receiver path.

Another interesting study is the benefits evaluation of using the proposed structure as a region reserved for the design of noisy circuits. The idea is to measure the noise propagated into the substrate, when generated, for example, by a NMOS transistor operating inside the field implant/pseudo deep nwell structure. The experience was carried out on the fabricated test structures, represented in Fig. 9. These test structures are geometrically identical to the ones shown in Figs. 7 b) to d). The difference is that the signals are now injected through an $n+$ implant (left), acting as the source or drain implant of an isolated NMOS, and collected by a p+ pick-up implant.

The experience was conducted with an Agilent E4438C Vector Signal Generator, acting as a "sinusoidal noise source", imposing $20 \mathrm{dBm}$ at $\mathrm{n}+$ noise port. An Agilent E4404B Spectrum Analyser ( $<6.7 \mathrm{GHz}$ ) was used to measure the spectral response of the signal collected at the $p+$ pick up. In this measurement the VNA was not used because its dynamic range is not large enough to characterize with accuracy the 
isolation achieved by the pseudo deep n-well structure. This happens because there are two series junction capacitances in the path of the noise, imposing a very large attenuation. The experimental results are shown in Fig. 10 , for $5 \mathrm{MHz}, 50$ $\mathrm{MHz}, 500 \mathrm{MHz}$ and $5 \mathrm{GHz}$. As can be seen, when the $\mathrm{n}+$ noisy implant is immersed in the proposed structure, the noise level reaches $-50 \mathrm{dBm}$, which corresponds to an improvement of more than $20 \mathrm{~dB}$ for frequencies up to $1 \mathrm{GHz}$, when compared to the classical $\mathrm{p}+$ guard-ring solution. This difference is then reduced at $5 \mathrm{GHz}$. These results indicate a clear advantage of designing the noisy blocks of the integrated system (high frequency digital and switch mode power management or amplifying circuits) inside the pseudo deep $\mathrm{n}$ well. In this way, the noise injected to the substrate is highly reduced. This, of course, is attained with no penalty in Silicon area and with no modification in the CMOS process normal flows.

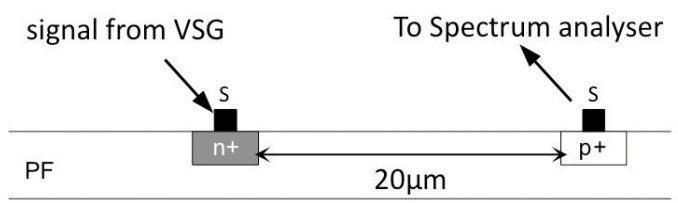

$$
\text { p-sub }
$$

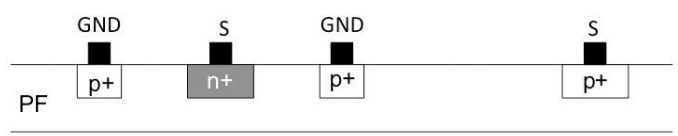

p-sub

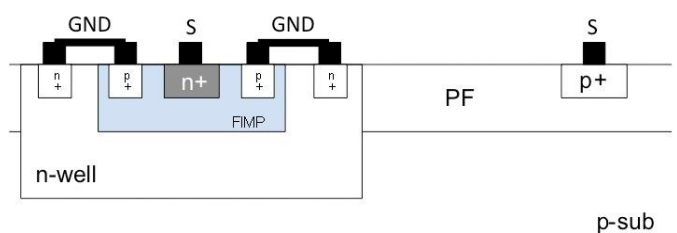

Fig. 9 - Test structures to evaluate noise injection into the substrate: a) cross section representation of the reference structure; $b$ ) the $n+$ implant surrounded by a classical $\mathrm{p}+$ guard-ring; c) the $\mathrm{n}+$ implant enveloped by the proposed field implant/pseudo n-well junction isolation structure.

\section{CONCLUSIONS}

It was successfully demonstrated throughout experiments on die the viability and the improvements of processing the field implant region inside the $\mathrm{n}$-well, in a single well standard CMOS process. The proposed method is purely based on layout mask manipulation and is fully CMOS compatible. In this way, the single n-well can also be used as a pseudo deep n-well, like in recent nodes of CMOS technology, to increase noise isolation.

According to experimental results up to $3 \mathrm{GHz}$, the proposed structure reduces the substrate noise propagation when compared with the classical $\mathrm{p}+$ guard-ring. This improvement exceeds $20 \mathrm{~dB}$ for low frequencies and a minimum of $4 \mathrm{~dB}$ at $3 \mathrm{GHz}$. Furthermore, experimental results also confirm that the performance of NMOS devices fabricated inside the pseudo deep n-well is similar to the process NMOS counterpart.

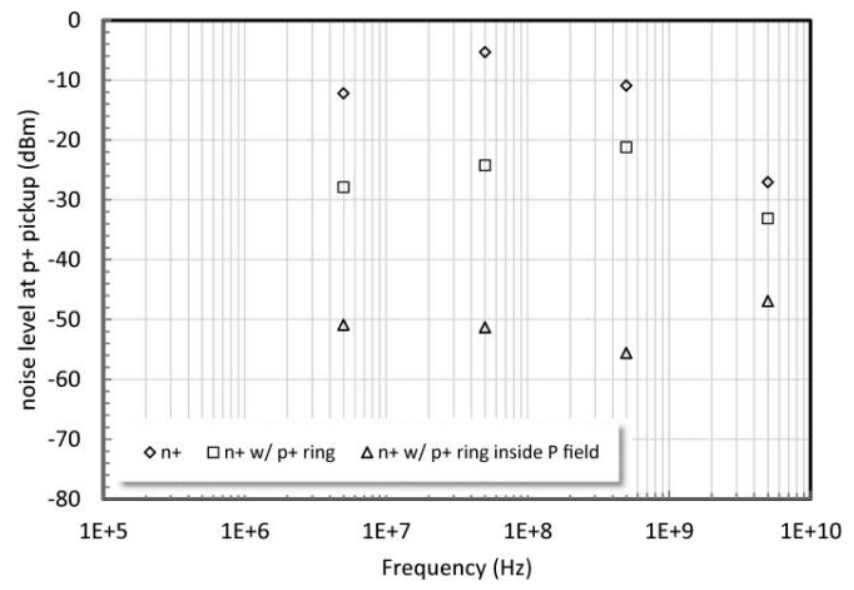

Fig. 10 - Experimental results of the noise level (in $\mathrm{dBm}$ ) measured at the $\mathrm{p}+$ pick-up (see Fig. 9).

The proposed methodology is accessible to any designer of mixed signal circuits since it is fully CMOS compatible. With the availability of the pseudo deep n-well, circuits' performance can be improved beyond the limits usually imposed by this low cost CMOS processes.

\section{ACKNOWLEDGEMENTS}

This work was funded by FCT projects UID/EEA/50008/2013 and PEst-OE/EEI/LA0008/2014.

\section{REFERENCES}

[1] J. Su, H. Hsu, S. Wong, C. Chang, T. Huang and J. Y. Sun, "Improving the RF Performance of $0.18 \mu \mathrm{m}$ CMOS With Deep n-Well Implantation", IEEE Electron Device Letters, V. 22, N. 10, pp. 481-483, October 2001.

[2] K. Chew, J. Zhang, K. Shao, W. B. Loh and S-. F. Chu, "Impact of Deep $\mathrm{N}$-well Implantation on Substrate Noise Coupling and RF Transistor Performance for Systems-on-a-Chip Integration", 32th European Solid-State Device Research Conference, ESSDERC2002, Firenze, Italy, pp. 251-254, September 2002.

[3] W. Yeh, S. Chen and Y. Fang, "Substrate Noise-Coupling Characterization and Efficient Suppression in CMOS Technology", IEEE Transactions on Electron Devices, V. 51, N. 5, pp. 817-819, May 2004.

[4] Y. Ogasahara, M. Hashimoto, T. Kanamoto and T. Onoye, "Measurement of Supply Noise Suppression by Substrate and Deep N-well in 90nm Process", IEEE Asian Solid-State Circuits Conference, Fukuoka, Japan, pp. 397-400, November 2008.

[5] Y. Wu, S. S. H. Hsu, K. K. W. Tan and Y. Su, "Substrate Noise Coupling Reduction in LC Voltage-Controlled Oscillators", IEEE Electron Device Letters, V. 30, N. 4, pp. 383-385, April 2009.

[6] D. Yang, Y. Ding and S. Huang, "A 65-nm High-Frequency Low-Noise CMOS-Based RF SoC Technology", IEEE Transactions on Electron Devices, V. 57, N. 1, pp. 328-335, January 2010. 
[7] J. Kang, D. Yu, Y. Yang and B. Kim, "Highly Linear $0.18 \mu \mathrm{m}$ CMOS Power Amplifier With Deep n Well Structure", IEEE Journal of Solid-State Circuits, V. 41, N. 5, pp. 1073-1080, May 2006.

[8] W. Sun, J. Wu, S. Lu, Y. Yi and L. Shi, "High-Voltage Power Integrated Circuit Technology Using Bulk-Silicon for Plasma Display Panels Data Driver IC", Microelectronics Engineering, Elsevier, V. 71, N. 1, pp. 112-118, January 2004.

[9] M. Knaipp, J. M. Park and V. Vescoli, "Evolution of a CMOS Based Lateral High Voltage Technology Concept", Microelectronics Journal, Elsevier, V. 37, N. 3, pp. 243-248, March 2006.

[10] W. Sun, Y. Yi and L. Shi, "A Novel Latch-Up Protection for BulkSilicon Scan Driver ICs of Shadow-Mask Plasma-Display Panel", IEEE Electron Device Letters, V. 28, N. 12, pp. 1135-1137, December 2007.

[11] H. Li, Q. Qian, H. Wu, W. Sun and L. Shi, "Low Cost Bulk Silicon CDMOS Technology and Enhanced dv/dt High Voltage Driver Circuit for PDP Data Driver IC", Microelectronics Journal, Elsevier, V. 40, N. 6, pp. 939-943, June 2009.

[12] Berk Camli, Baykal Sarioglu, and Arda D. Yalcinkaya, "Photodiodes for Monolithic CMOS Circuit Applications", IEEE Journal of Selected Topics in Quantum Electronics, V. 20, N. 6, Nov./Dec. 2014.

[13] Fsfsdsf Fernando de Souza Campos, Naser Faramarzpour, Ognian Marinov, M. Jamal Deen, and Jacobus W. Swart, "Photodetection With GateControlled Lateral BJTs from Standard CMOS Technology", IEEE Sensors Journal, V. 13, N. 5, pp. 1554-1563, May 2013.

[14] fsdff Berkehan Ciftcioglu, Lin Zhang, Jie Zhang, John R. Marciante, Jonathan Zuegel, Roman Sobolewski, and Hui Wu, "Integrated Silicon PIN Photodiodes Using Deep N-Well in a Standard $0.18 \mu \square \mathrm{m} \quad \mathrm{CMOS}$ Technology", Journal of Lightwave Technology, V. 27, N. 15, pp. 3303-3312, Aug. 1, 2009.

[15] H. J. Quaresma, P. Mendonça dos Santos, A. Cruz Serra, "An equivalent doping profile for CMOS substrate characterization", Solid State Electronics, Elsevier, V. 79, pp. 185-191, Jan. 2013.

[16] D. Kosaka, M. Nagata, Y. Hiraoka, I. Imanishi, M. Maeda, Y. Murasaka and A. Iwata, "Isolation Strategy Against Substrate Coupling in CMOS Mixed Signal/RF Circuits", Symposium on VLSI Circuits, 2005, Kyoto, Japan, pp. 276- 279, June 2005. 